\title{
Sudden cardiac death in a young, male patient with rapidly progressing systemic sclerosis
}

\author{
Anna Włudarczyk ${ }^{1}$, Maria Ignacak-Popiel ${ }^{1}$, Jacek Górka ${ }^{1}$, Wojciech Szczeklik ${ }^{1}$, \\ Magdalena Ulatowska-Białas ${ }^{2}$, Jacek Musiał ${ }^{1}$ \\ ${ }^{1} 2^{\text {nd }}$ Department of Internal Medicine, Jagiellonian University Medical College, Krakow, Poland \\ ${ }^{2}$ Department of Pathology, Jagiellonian University Medical College, Krakow, Poland
}

A 22-year-old man was admitted to the Immunology Department because of rapidly progressive skin hardening. In the previous two years he was treated with methylprednisolone and azathioprine for autoimmune hepatitis. On admission the patient presented with massive hardening of the skin of the lower part of the face (causing severe microstomia), and skin of the neck, chest, abdomen, and upper limbs, lower limbs were involved to a lesser extent. The involved skin was hardened, shiny, and dry with exfoliating epidermis. Capillary refill was delayed. The patient was cachectic and reported difficulty with swallowing solid food. He also complained of hoarseness and a sore throat. His speech was slurred. A throat examination was impossible to perform due to microstomia. Family history was positive for liver and testicular cancer. The skin started to harden four months prior to admission, and since then the patient was thoroughly screened to exclude any neoplasm. Gastroscopy revealed inflammatory infiltrates in the stomach; colonoscopy was negative. Urological examination including testicular ultrasound was normal. Chest computed tomography revealed multiple ground glass opacities. Cultures from the material collected during bronchoscopy showed Pseudomonas aeruginosa. Antibiotics were started. Lung tests revealed reversible airway obstruction and mild pulmonary hypertension. He was then consulted by the cardiologist, who did not find signs of right ventricular overload or cardiac failure and suggested further observation. On admission the patient's vital signs were within normal range (heart rate $95 \mathrm{bpm}$, blood pressure $120 / 80 \mathrm{mmHg}$, haemoglobin oxygen saturation $96 \%$ ) and did not change significantly during hospitalisation. He presented mild anaemia (haemoglobin $11.6 \mathrm{~g} / \mathrm{dL}$ ), elevated platelet count $\left(402 \times 10^{3} / \mu \mathrm{L}\right)$, monocytes $\left(1.56 \times 10^{3} / \mu \mathrm{L}\right)$, and eosinophils $\left(0.89 \times 10^{3} / \mu \mathrm{L}\right)$. Serum albumin level and cholinesterase activity were slightly decreased. International normalised ratio was 1.18. C-reactive protein and lactate dehydrogenase were elevated. Polyclonal hypergammaglobulinaemia was present. Electrocardiogram was impossible to obtain because of severe skin hardening causing multiple artefacts. Heart ultrasound showed traces of fluid in the pericardium. Left ventricular ejection fraction was $\sim 65 \%$. There was no thickening of the heart muscle or hypokinesis. Pulmonary artery systolic pressure was estimated around $40 \mathrm{mmHg}$ using continuous Doppler wave method. Barium swallow examination of the oesophagus revealed dysmotility. Anti-nuclear antibodies were positive in immunofluorescence test (titre 1:320) with a speckled pattern, but specific identification was negative on immunoblot. Skin and muscle biopsy showed fibrosis of the skin and fascia with mild inflammation in the epimysium. The patient was diagnosed with diffuse systemic sclerosis and received two 250-mg pulses of methylprednisolone followed by $1 \mathrm{mg} / \mathrm{kg}$ body weight daily IV dose. Topical steroids were also applied. The patient initially reported improvement in skin tension. On the $14^{\text {th }}$ day of hospitalisation the nurse found the patient unreactive and alerted the resuscitation team. Sudden cardiac arrest with asystole as a presenting rhythm was recognised. Despite long-lasting (over $1 \mathrm{~h}$ ) cardiopulmonary resuscitation with cricothyrotomy procedure (microstomia made intubation difficult) and multiple adrenaline doses the patient died. Autopsy was performed, but

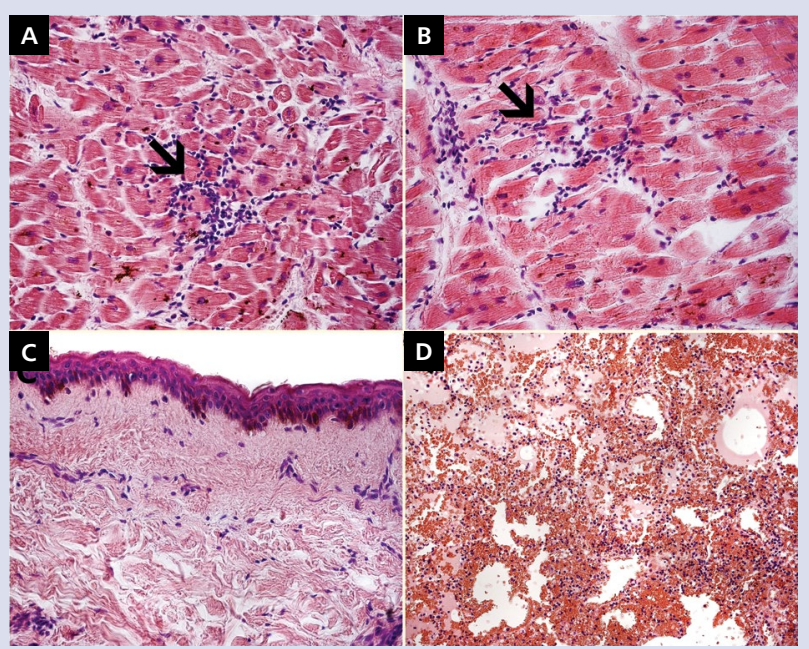

Figure 1. A, B. Myocarditis. Lymphocytic infiltrate (arrows) leading to cardiomyocytes damage $(\times 40)$; C. Skin sample. Epidermis thinning, lack of skin appendages and thick collagen fibres under epidermis $(\times 20)$; D. Lung sample. Hyperemia, swelling and focal haemorrhages $(\times 20)$ macroscopic examination did not reveal the cause of death. However, microscopic examination of the cardiac muscle showed massive lymphocyte infiltrates with cardiomyocyte damage typical for severe myocarditis (Fig. 1A, B). Fibrosis of the skin was confirmed (Fig. 1C) together with lung fibrosis (Fig. 1D). Based on the autopsy findings, the most probable cause of death was clinically asymptomatic, rapidly progressive myocarditis. Systemic sclerosis is a rare autoimmune disease that affects mostly women in their fifties [1]. It has heterogeneous presentations and can involve the skin, muscles, joints, vessels, and almost any internal organ [2]. Heart involvement is associated with a poor prognosis [3]. Although typically the disease progresses slowly, it can have a rapid course. Cardiac involvement should always be considered even when initially asymptomatic.

\section{References}

1. Chifflot $\mathrm{H}$, Fautrel B, Sordet $\mathrm{C}$, et al. Incidence and prevalence of systemic sclerosis: a systematic literature review. Semin Arthritis Rheum. 2008; 37(4): 223-235, doi: 10.1016/j.semarthrit.2007.05.003, indexed in Pubmed: 17692364

2. Pattanaik D, Brown M, Postlethwaite B, et al. Pathogenesis of Systemic Sclerosis. Front Immunol. 2015; 6, doi: 10.3389/fimmu.2015.00272.

. Elhai M, Meune C, Avouac J, et al. Trends in mortality in patients with systemic sclerosis over 40 years: a systematic review and meta-analysis of cohor studies. Rheumatology (Oxford). 2012; 51(6): 1017-1026, doi: 10.1093/rheumatology/ker269, indexed in Pubmed: 21900368.

\section{Address for correspondence:}

Anna Włudarczyk, MD, $2^{\text {nd }}$ Department of Internal Medicine, Jagiellonian University Medical College, ul. Skawińska 8, 31-066 Kraków, Poland, e-mail: anna.wludarczyk@uj.edu.pl

Conflict of interest: none declared

Kardiologia Polska Copyright (c) Polish Cardiac Society 2018 